Paediatr Paedolog 2018 · 53:278-283 https://doi.org/10.1007/s00608-018-0627-9 Online publiziert:5. November 2018 (c) Der/die Autor(en) 2018

CrossMark

Saskia B. Wortmann ${ }^{1,2}$. Johannes Spenger ${ }^{1}$ Martin Preisel ${ }^{1}$. Johannes Koch ${ }^{1}$ • Christian Rauscher ${ }^{1}$. Ingrid Bader ${ }^{3}$. Johannes A. Mayr ${ }^{1}$ - Wolfgang Sperl ${ }^{1}$

${ }^{1}$ Universitätsklinik für Kinder- und Jugendheilkunde, Paracelsus Medizinische Privatuniversität, Salzburg, Österreich

${ }^{2}$ Institut für Humangenetik, Technische Universität München, München, Deutschland

${ }^{3}$ Division für klinische Genetik, Universitätsklinik für Kinder- und Jugendheilkunde, Paracelsus

Medizinische Privatuniversität, Salzburg, Österreich

\title{
Next-Generation-Sequenzierung - Next-Generation-Qualität in der Pädiatrie
}

Die zunehmende Verfügbarkeit der $\mathrm{Me}$ rung ist dabei, die Diagnostik in der Kinder- und Jugendmedizin grundlegend zu verändern. Schon immer gehörte die Versorgung von Kindern mit angeborenen Erkrankungen und komplexen Fehlbildungssyndromen zum pädiatrischen Alltag. Allerdings ist es erst jetzt zunehmend möglich, überhaupt eine (genetische) Diagnose zu stellen. Die nahezu explodierende Anzahl an GenKrankheits-Assoziationen eröffnet viele Möglichkeiten, die zugrundeliegende $\mathrm{Pa}$ thophysiologie besser zu verstehen und - in einer kleinen, aber steigenden Zahl von Fällen - auch gezielt therapeutisch einzugreifen. Auch werden Diagnosen jetzt häufig schneller und in einem deutlich früheren Krankheitsstadium gestellt, was nochmals andere Aspekte mit sich bringt, wie z.B. Entscheidungen über Therapieanpassung an $\mathrm{zu}$ erwartende Krankheitssymptome in einem frühen Stadium der Erkrankung. Dieser Beitrag gibt einen kurzen Überblick über die am häufigsten angewendete Methode der Next-Generation-Sequenzierung in der Pädiatrie, der Whole-Exom-Sequenzierung (WES). Des Weiteren beleuchten wir, welche Veränderungen diese $\mathrm{Me}$ thode für das behandelnde Team und die Familien mit sich bringen und bringen werden. Wie sieht sie aus, diese Next-

Literatur bei den Verfassern
Generation-Qualität in der Kinder- und Jugendheilkunde?

WES-Daten werden in allen Laboren nach ähnlichen Algorithmen ausgewertet. Die Datenfilterung beruht dabei auf zwei Grundannahmen:

\section{Genetische Erkrankungen in der Kinder- und Jugendheilkunde}

Monogene Erkrankungen sind Erkrankungen, die durch pathogene Varianten (Mutationen) in einem einzelnen Gen verursacht werden. Einige Beispiele sind in $\bullet$ Tab. 1 aufgeführt. Es sind seltene Erkrankungen (Inzidenz $<1: 2000$ ), betreffen in ihrer Gesamtheit aber viele pädiatrische Patienten. Außerdem sind sie die häufigste Ursache für Todesfälle im 1. Lebensjahr.

\section{Whole-Exom-Sequenzierung}

Für die WES werden etwa 3-5 ml EDTABlut (bei Säuglingen genügen $2 \mathrm{ml}$ ) zur DNA-Extraktion aus Leukozyten benötigt. Die proteinkodierenden, exonischen DNA-Abschnitte werden zunächst angereichert und in der Folge mehrfach parallel sequenziert. Danach werden die erhaltenen Sequenzdaten mit einer Referenzsequenz verglichen. Abweichungen von der Referenzsequenz werden als Varianten bezeichnet. Im Mittel finden sich bei jedem Menschen etwa 30.000 Varianten. Die Mehrzahl dieser Varianten ist nicht krankheitsrelevant und es bedarf mehrerer Filterschritte, um die pathogene(n) Variante(n) für eine monogene Erkrankung zu identifizieren. 


\begin{tabular}{|c|c|c|c|}
\hline Typische Leitsymptome & $\begin{array}{l}\text { Beispielhafte(s) Krankheits- } \\
\text { bild(er) }\end{array}$ & $\begin{array}{l}\text { Anzahl }(n) \text { der in OMIM geliste- } \\
\text { ten Gen-Krankheits-Assoziationen } \\
\text { (26. Juli 2018) nach Suchbegriff }\end{array}$ & $\begin{array}{l}\text { Anzahl bekannter } \\
\text { Krankheits- und } \\
\text { Kandidatengene }\end{array}$ \\
\hline $\begin{array}{l}\text { Entwicklungsverzögerung } \pm \\
\text { Intelligenzminderung }\end{array}$ & Sehr viele & „Developmental delay“ $n \sim 5294$ & $>565$ \\
\hline Muskelschwäche \pm CK-Erhöhung & $\begin{array}{l}\text { Myopathie (z. B. Muskeldystrophie } \\
\text { Duchenne) }\end{array}$ & "Myopathy" $n \sim 368$ & $>70$ \\
\hline Bewegungsstörung (z. B. Ataxie, Dystonie) & Sehr viele & "Movement disorder" $n \sim 3925$ & $>65$ \\
\hline Epilepsie & $\begin{array}{l}\text { Sehr viele (z. B. Othahara- oder } \\
\text { Dravet-Syndrom) }\end{array}$ & „Epilepsy“ $n \sim 498$ & $>269$ \\
\hline Schwerhörigkeit & Sehr viele (z. B. Pendred-Syndrom) & "Deafness" $n \sim 1697$ & $>142$ \\
\hline Verminderte Sehfähigkeit & Sehr viele & „Blindness" $n \sim 310$ & $>200$ \\
\hline \multicolumn{4}{|c|}{$\begin{array}{l}\text { "Beschränkt auf Ergebnisse mit phänotypische Beschreibung, molekulargenetische Basis bekannt; enthält auch nicht monogene Krankheitsbilder (z. B. } \\
\text { Mikrodeletionssyndrome) }\end{array}$} \\
\hline
\end{tabular}

kann. Mutationen in 3941 verschiedenen Genen, also etwa einem Fünftel der bekannten Gene des Menschen, sind ursächlich für diese Krankheiten.

Die klinische WES-Analyse beschränkt sich auf die Suche nach potenziell krankheitsverursachenden Varianten in Genen, die in OMIM gelistet werden (Krankheitsgene). Ist eine beim Patienten gefundene Variante hier als krankheitsauslösend beschrieben, wird sie als pathogen eingestuft und berichtet. Finden sich unbekannte Varianten in einem Gen, helfen Softwareprogramme bei der Einschätzung der Pathogenität. Die Variante kann als wahrscheinlich pathogen oder als Variante unklarer Signifikanz (VUS) berichtet werden. Hierbei wiegt selbstverständlich stark, ob der klinische Phänotyp des Patienten mit den in der Fachliteratur beschriebenen Krankheitssymptomen übereinstimmt. Dies unterstreicht die Wichtigkeit einer guten klinischen Phänotypisierung (und die Mitteilung dieser Information an das WES-Auswerte-Team). Ebenso wichtig ist die Stammbaumerhebung/ Familienanamnese, da für die verschiedenen Krankheitsgene unterschiedliche Erbgänge typisch sind (autosomal dominant, autosomal rezessiv, X-gebunden, mitochondrial).

Finden sich in den OMIM-Genen keine bekannten Varianten, kann die Analyse auf alle menschlichen Gene ausgedehnt werden, jedoch handelt es sich dann im strengen Sinn nicht mehr um eine klinische WES-Analyse, sondern um eine Untersuchung mit gewis- sem Forschungscharakter. Varianten, die hierbei als potenziell krankheitsverursachend eingeschätzt werden, bedürfen idealerweise funktioneller Bestätigung (biochemische Untersuchungen z.B. in kultivierten Hautfibroblasten) oder zumindest des Nachweises über denselben Phänotyp in mehreren Patienten. So kann dann eine neue Gen-KrankheitsAssoziation hergestellt werden.

Generell ermöglichen die für die Exomsequenzierung generierten Daten auch eine gezielte Auswertung bezüglich „copy number variation" (CNV), z.B. Deletionen, und Varianten in der mitochondrialen DNA. Dies ist aber abhängig von der Erfahrung des ausführenden Labors, gegebenenfalls empfiehlt sich die zusätzliche Durchführung eines konventionellen Arrays.

Idealerweise sollten nicht nur die Sequenzdaten des Kinds vorliegen, sondern auch eine Analyse als Eltern-Kind-Trio (TRIO-WES) erfolgen. Diese Analyse ist insbesondere wichtig bei genetischen Erkrankungen, die nicht vererbet werden, sondern de novo entstanden sind (häufig z. B. bei Epilepsie, Entwicklungsverzögerung oder Intelligenzminderung). Generell ist eine Trio-WES leichter auszuwerten, da die elterlichen Daten als Vergleich vorliegen und sofort nachvollziehbar ist, ob z. B. bei rezessiven Erkrankungen 2 Varianten beim Kind auch tatsächlich biallelisch vorliegen. Dieser Zeitaspekt sollte insbesondere bei kritisch kranken Kindern berücksichtigt werden.

Mit den oben genannten Schritten und in der interdisziplinären Zusam- menschau von Klinik/Phänotyp und der Möglichkeit funktioneller biochemischer Diagnostik gelingt es, je nach Indikation, in bis zu $50 \%$ aller Fälle eine genetische Diagnose zu stellen (• Abb. 1).

Dass eine Diagnosestellung nicht immer gelingt, kann verschiedene Gründe haben:

- Krankheitsauslösende Varianten können nicht erkannt werden, wenn z. B. das betroffene Gen noch nicht als Krankheitsgen beschrieben ist oder der klinische Phänotyp vom bisher beschriebenen abweicht. Daher empfiehlt sich eine regelmäßige Reanalyse der Exomsequenzierungsdaten z. B. im 2-jährlichen Abstand.

- Varianten können in Abschnitten des Genoms liegen, die bei der WES nicht erfasst werden (etwa 1-2\% aller exonischen Abschnitte, intronische Abschnitte, regulatorische Elemente). Daher kann bei negativer WES eine Whole-Genome-Sequenzierung sinnvoll sein.

- Bestimmte genetische Erkrankungen (z.B. Trinukleotidexpansionen wie beim Fragilen-X-Syndrom oder der Friedreich'schen Ataxie) können mit WES ebenfalls nicht erfasst werden und erfordern eine separate Abklärung.

- Auch werden chromosomale Auffälligkeiten (z. B. Ringchromosom, komplexe chromosomale Aberrationen) nicht erfasst, sodass die WES die Karyotypisierung nicht ersetzen kann. 
- Bei Verdacht auf Erkrankungen aufgrund von mitochondrialen DNA-Veränderungen ist zu beachten, dass eine Gewebespezifität vorliegen kann und die WES aus Blut die Veränderung nicht erfassen kann. Hier empfiehlt sich eine separate Analyse der mitochondrialen DNA, insbesondere auch bei Verdacht auf mitochondriale Deletion(en), da diese ebenfalls in der WES nicht detektiert werden können.

\section{Phänotypisierung - wie viel Diagnostik ist vor der WES notwendig und sinnvoll?}

Die Auswertung der WES-Daten ist abhängig von der Qualität der vorangegangenen Phänotypisierung, allerdings kann die Exomsequenzierung bei sehr unspezifischen klinischen Symptomen wie globale Entwicklungsverzögerung auch zu Befunden führen, die eine nachgeordnete Phänotypisierung erfordern ("reverse phenotyping“). Eine sorgfältige Erhebung der Anamnese, der Familienanamnese inklusive eines Stammbaums über drei Generationen sowie eine ausführliche klinisch-neurologische Untersuchung sind selbstverständlich vorher durchzuführen. Der anfordernde Arzt muss danach gut abwägen, welche (invasiven) Untersuchungen vor einer Exomsequenzierung gerechtfertigt sind, da sie entweder für eine Behandlungsentscheidung essenziell sind oder bei der Auswertung der Exomsequenzierungsdaten helfen könnten. Unzählige ungerichtete Screeninguntersuchungen können so vermieden werden und wiegen die (relativ) hohen Kosten der WES auf, wie mehrere Studien gezeigt haben. Aufgrund der fehlenden Detektionsmöglichkeit des Fragilen-X-Syndroms sollte diese Untersuchung ergänzt werden. Ebenso sollte die CNV-Analyse mithilfe von Arrays aus oben geschilderten Gründen gegebenenfalls ergänzt werden. Somit wird immer deutlicher, dass die neuen genetischen Untersuchungsmethoden, wenn sie gezielt eingesetzt werden, eigentlich ökonomisch von klarem Vorteil sind. Das wird auch zunehmend von den Krankenkassen so gesehen. Zudem ist eine zu breite und unkritische Anwendung von WES durch die Etablierung und Filterfunktion der Medizinisch-genetischen Zentren in Österreich eher unterbunden. Pragmatischerweise erscheint also ein Geneticsfirst-Ansatz medizinisch und ökonomisch gerechtfertigt.

\section{Wie kommt der Patient zu einer Diagnose - und wie kommt die Diagnose wieder zum Patienten?}

Die Exomsequenzierung an sich und die gefundenen seltenen Erkrankungen erfordern ein enormes Detailwissen, das eine meist internationale Teamarbeit über die Spezialisierungsgrenzen hinaus erfordert.

Wie bei der Durchführung aller Untersuchungen, sollte auch eine WES immer als Ziel haben, dass das Untersuchungsergebnis eine für den Patienten bzw. dessen Eltern relevante Information beinhaltet oder für die Behandlung entscheidend ist. Und genau hier liegt sowohl die Herausforderung, als auch die Chance. Die - Abb. 1 zeigt, wie eine holistische Betreuung von Kindern (z.B. mit Entwicklungsverzögerung) mit seltenen Erkrankungen an einem Kinderzentrum aussehen könnte.

Die Hauptaufgabe des Kinderzentrums ist es, so schnell wie möglich und mit so wenigen (invasiven) Untersuchungen wie möglich eine fundierte Diagnose zu stellen und die Betreuung bzw. Behandlung des Patienten zu koordinieren. Jeder, der mit der Betreuung dieser Kinder beschäftigt ist, weiß, dass die Patienten häufig eine lange Odyssee mit vielen Arztbesuchen und Untersuchungen hinter sich haben, bevor eine Diagnose gestellt wird. Ein Kind mit Entwicklungsverzögerung wird manchmal zuerst dem Neuropädiater, manchmal dem Humangenetiker und manchmal dem Spezialisten für angeborene Stoffwechselerkrankungen vorgestellt. Immer wieder stellen sich solche Patienten auch in der Akutambulanz mit einer Einweisung zur stationären Abklärung vor.

Um die Zeit optimal zu nutzen und die Anzahl der Arztkontakte bzw. Untersuchungen (und somit auch Kosten) zu minimieren, empfehlen wir, alle Kin-
Paediatr Paedolog 2018 $\cdot 53: 278-283$

https://doi.org/10.1007/s00608-018-0627-9

○ Der/die Autor(en) 2018

S. B. Wortmann · J. Spenger · M. Preisel · J. Koch · C. Rauscher · I. Bader · J. A. Mayr . W. Sperl

\section{Next-Generation- Sequenzierung - Next- Generation-Qualität in der Pädiatrie}

\section{Zusammenfassung}

Die Exomsequenzierung ermöglicht es, bei immer mehr Patienten mit z. B. Entwicklungsverzögerung den zugrundeliegenden genetischen Defekt zu diagnostizieren. Hierdurch vermehrt sich unser Wissen zur Pathophysiologie und es eröffnen sich einer kleinen, aber steigenden Zahl von Patienten gezielte Therapieoptionen. Dieser Beitrag beleuchtet die Möglichkeiten und Limitierungen der Methode und ihre Auswirkungen auf den Alltag des Facharztes für Kinder- und Jugendheilkunde.

Schlüsselwörter

Next-Generation-Sequenzierung · Exomsequenzierung · Entwicklungsverzögerung · Intelligenzminderung · Genetik

\section{Next-Generation Sequencing: Next-Generation Quality in Pediatrics}

\section{Abstract}

Exome sequencing enables diagnosis of the underlying genetic defect in a growing number of patients with, for example, developmental delays. This increases our knowledge of the underlying pathophysiology and opens up therapeutic intervention to a small but growing number of patients. This article examines the opportunities and limitations of exome sequencing and its influence on daily pediatric practice.

\section{Keywords}

Next-generation sequencing · Exome sequencing · Developmental delay · Menta retardation. Genetics 


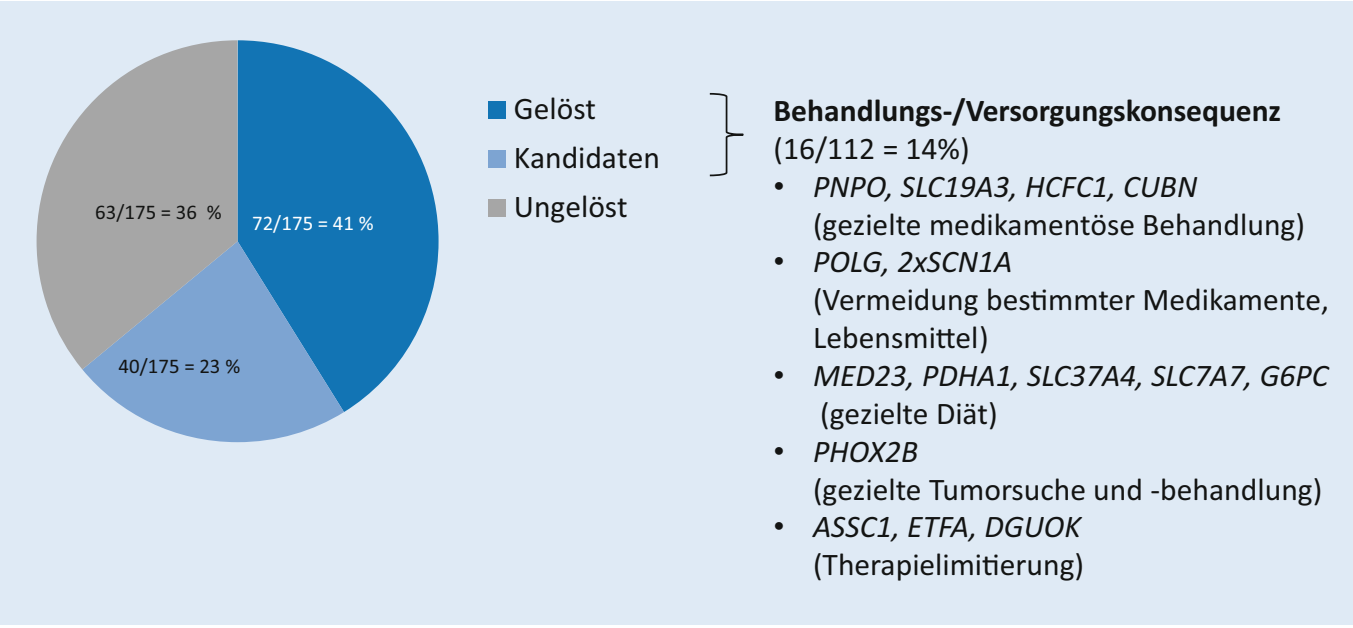

Abb. $1 \triangleleft$ Ergebnisse und Auswirkungen auf die weitere Behandlung aus 175 Exomsequenzierungen der Universitätsklinik für Kinder-und Jugendheilkunde Salzburg

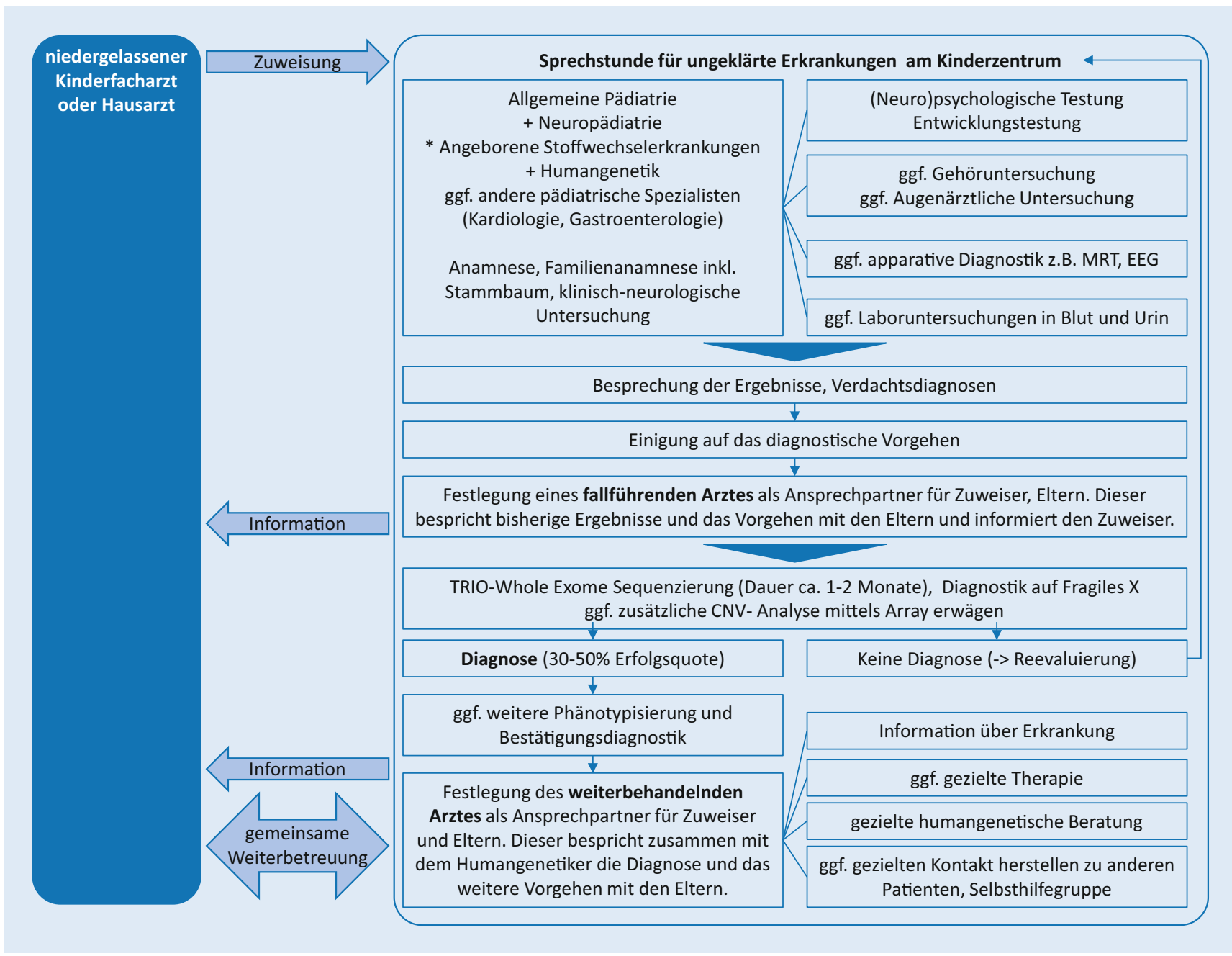

Abb. $2 \Delta$ Ablaufschema Evaluierung eines entwicklungsverzögerten Kinds an der Universitätsklinik für Kinder- und Jugendheilkunde Salzburg. CNV „"copy number variation“; EEG Elektroenzephalographie; MRT Magnetresonanztomographie 
der in einer gemeinsamen Sprechstunde bzw. im Rahmen einer tagesstationären Aufnahme der genannten Disziplinen zu sehen. Wie in - Abb. 1 gezeigt, können dann auch zeitnah ergänzende Untersuchungen durchgeführt werden, insbesondere, wenn sie eine Behandlungsrelevanz haben. Genauso ist hier die Abklärung von Hör- bzw. Sehverlust zu erwähnen, da sich hierbei in der Regel Behandlungsmöglichkeiten bieten. Weiterhin sollte ein fallführender Arzt benannt werden, der während des diagnostischen Prozesses die Koordination übernimmt und Ansprechpartner für Zuweiser und die Eltern ist. Wird innerhalb dieser Abklärung eine Diagnose erzielt, sollte festgelegt werden, welcher Arzt bzw. welche Fachrichtung die weitere Betreuung des Patienten übernimmt und in der weiteren Folge sollte ein gemeinsames pädiatrisch-genetisches Diagnosegespräch stattfinden. Der fallführende Arzt hat nun vielfältige Aufgaben: Literaturrecherche, Information des zuweisenden Kinderfacharztes oder Hausarztes, Information der Eltern, falls gewünscht auch Herstellen eines Kontakts zu anderen Patienten, Überweisung an ein auf die gefundene Erkrankung spezialisiertes Kinderzentrum, Evaluation gezielter Therapiemöglichkeiten (• Abb. 2), z.B. im Rahmen von Studien.

Patienten, bei denen keine Diagnose gestellt wurde, sollten in 1- oder 2-jährlichem Abstand erneut vorgestellt werden.

Die Bündelung des Wissens und die standardisierte Patientenversorgung erlauben es, auch Patienten mit seltenen Erkrankungen gemäß „evidence-based best practice“ zu behandeln und eröffnet somit eine Next-Generation-Qualität in der Kinder- und Jugendheilkunde.

\section{Korrespondenzadresse}

\section{Saskia B. Wortmann}

Universitätsklinik für Kinder- und Jugendheilkunde, Paracelsus Medizinische Privatuniversität

Salzburg, Österreich

s.wortmann@salk.at

Funding. Open access funding provided by Paracelsus Medical University.

\section{Einhaltung ethischer Richtlinien}

Open Access Dieser Artikel wird unter der Creative Commons Namensnennung 4.0 International Lizenz (http://creativecommons.org/licenses/by/4.0/deed. de) veröffentlicht, welche die Nutzung, Vervielfäl-

Interessenkonflikt. S.B. Wortmann, J. Spenger, M. Preisel, J. Koch, C. Rauscher, I. Bader, J.A. Mayr und W. Sperl geben an, dass kein Interessenkonflikt besteht. in jeglichem Medium und Format erlaubt, sofern Sie den/die ursprünglichen Autor(en) und die Quelle ordnungsgemäßnennen, einen Link zur Creative Com-

Dieser Beitrag beinhaltet keine von den Autoren durchgeführten Studien an Menschen oder Tieren. vorgenommen wurden.

\section{Anhang}

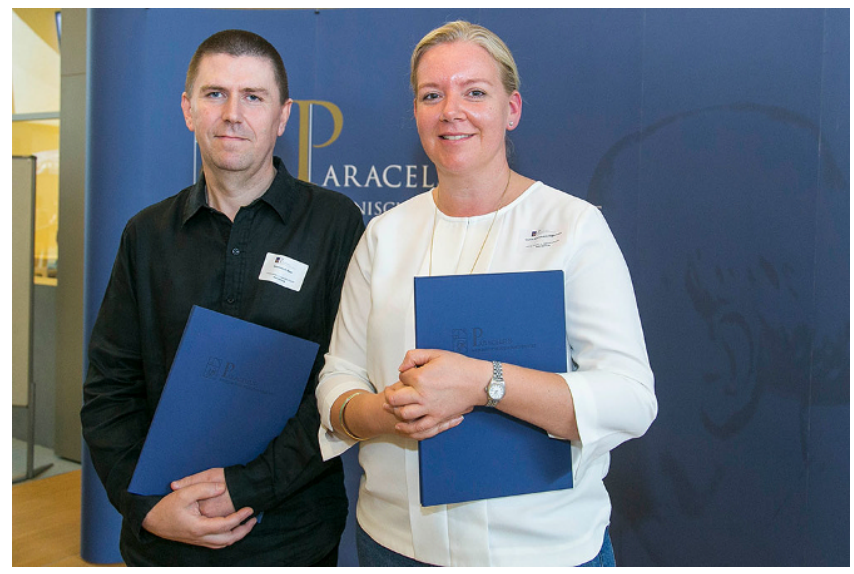

Abb. 3 ॥ Die Paracelsus Medizinische Privatuniversität zeichnete Ende Juni im Rahmen des Science Get Togethers Priv.-Doz. Dr. med. Dr. Saskia Wortmann als Forscherin des Jahres (Kategorie Klinische Fächer) und Priv.-Doz. Dipl.-Ing. Dr. Johannes Mayr (Kategorie Theoretische Fächer) als Forscher des Jahres aus. Beide arbeiten und forschen an der Universitätsklinik für Kinder- und Jugendheilkunde Salzburg (๑ PMU/wildbild) 
Hier steht eine Anzeige.

\section{曾 Springer}

